\title{
lip, a human gene detected by transfection of DNA from a human liposarcoma encodes a protein with homology to regulators of small G proteins
}

\author{
HELEN PATTERSON, ${ }^{1,2}$ SANDRA GILL, ${ }^{1}$ HARRY BENJAMIN, ${ }^{1}$ PHILIP J. MITCHELL ${ }^{2}$ \\ \& COLIN S. COOPER ${ }^{1}$
}

Section of ${ }^{1}$ Molecular Carcinogenesis and ${ }^{2}$ Cell Biology and Experimental Pathology, Institute of Cancer Research, Surrey, UK

\begin{abstract}
Purpose/Method. Transfection experiments have been used to identify activated oncogenes in a wide variety of tumour types. Here we describe the use of transfection experiments utilizing DNA from a human pleomorphic liposarcoma to identify a novel gene, designated lip which maps to chromosome 19.

Results. lip was expressed in all sarcoma cell lines examined and a wide variety of normal tissues. Sequencing of cDNAs prepared from transcripts of the normal lip gene indicates that lip is predicted to encode a 966 amino acid protein with a region of homology to proteins such as $v a v, d b l, l b c$ and $e c t-2$ which act as GDP-GTP exchange factors for the RAS superfamily of small GTP-binding proteins, and the N-terminal 830 amino acids are identical to the recently identified gene p115-RhoGEF, an exchange factor for RHOA. In transfectants, lip has undergone a rearrangement which results in C-terminal truncation of the predicted LIP protein. However, we failed to detect this alteration in the primary liposarcoma used in the original transfection experiments, or in other sarcoma specimens examined.

Discussion. When considered together, these observations suggest that transforming lip sequences represent an alternatively spliced form of pll5-RhoGEF that is activated for transformation by C-terminal truncation during transfection, and is not widely involved in sarcoma development.
\end{abstract}

Key words: transfection, soft tissue sarcoma, nucleotide exchange factor.

\section{Introduction}

Transfection of DNA into NIH3T3 mouse fibroblasts has been used to survey a wide variety of human tumours for the presence of transforming oncogenes. Although the majority of the genes detected by this assay are activated ras genes, a number of other genes including met, ${ }^{1}$ ret, trk $^{3}{ }^{3}$ mas $^{4}, \mathrm{dbl}^{5}{ }^{5} \mathrm{raf}^{6}{ }^{6}$ hst $^{7}{ }^{7}$ vav, ${ }^{8}$ ufolaxl, ${ }^{9,10}$ $e c t-2^{11}$ and $l b c^{12}$ have also been identified. These genes encode proteins of several functional classes, including growth factors, transmembrane receptors with tyrosine kinase activity. non-receptor serine-threonine kinases and regulators of small GTP-binding proteins, all of which are thought to play a role in intracellular signalling pathways which regulate cellular proliferation.

In an attempt to identify oncogenes activated in human soft tissue tumours, DNA from 29 sarcomas was examined for the ability to transform NIH3T3 cells. ${ }^{13}$ These studies identified an activated $k$-ras gene in a leiomyosarcoma, and a novel activated gene following transfection of DNA from a pleomorphic liposarcoma. Genomic fragments of this novel gene, designated lip, were cloned by screening a genomic library prepared using DNA from a lip secondary transfectant with a human $a l u$-repeat probe. Repeat-free subclones of these genomic clones have been used to demonstrate that this gene maps to chromosome 19 and is expressed as a $3.0-\mathrm{kb}$ transcript in primary and secondary lip transfectants. ${ }^{13}$

cDNAs corresponding to the normal lip gene have now been cloned from a normal fibroblast cDNA library and through sequencing analysis were found to encode a protein with regions of homology to proteins such as exchange factors for small GTPbinding proteins. In addition the $\mathrm{N}$-terminal 830 amino acids are almost completely identical to the recently cloned p115-RhoGEF. ${ }^{14}$ Sequencing of cDNA clones isolated from a primary transfectant 
library indicate that $3^{\prime}$ sequences are lost during its activation.

\section{Materials and Methods}

\section{$5^{\prime}$ RACE polymerase chain reaction analysis}

Cytoplasmic RNA was extracted from subconfluent cultures of MCF-7 cells. ${ }^{15} 5^{\prime}$ RACE (rapid amplification of cDNA ends) was performed as recommended using the 5' RACE System (Gibco BRL), with the exception that RNA was reverse transcribed using random hexamer primers (Pharmacia). Polymerase chain reaction (PCR) primers were as follows: first round of amplification GAGTTTGTCTCCAGCTCG, second round of amplification CTCAAAATCCTCATCCTCAGC.

\section{Preparation and screening of cDNA libraries}

cDNA clones corresponding to the normal LIP gene were obtained by screening a randomly primed HT1080 cDNA library ${ }^{16}$ and an oligo-dT-primed M426 human fibroblast cDNA library ${ }^{17}$ Clones corresponding to the transfected gene were obtained from a primary transfectant cDNA library constructed as follows. RNA was extracted from subconfluent cultures of transfectant cells, ${ }^{18}$ and poly $(\mathrm{A})^{+}$RNA selected using oligo-(dT)-cellulose (Poly(A) Quik Kit, Stratagene). The cDNA library was constructed in the lambda ZAP II vector (Stratagene) using the ZAP II cDNA synthesis kit (Stratagene). All three libraries were screened using Biodyne hybridization membranes (Pall-Biodyne) as previously described. ${ }^{16}$

\section{cDNA sequencing}

Partially deleted subclones of the cDNA inserts were generated using exonuclease III and mung bean nuclease (Stratagene), and these subclones sequenced by the dideoxy chain termination method $^{19}$ using the Sequenase Version 2.0 sequencing kit (United States Biochemicals).

Alternatively, primary transfectant poly(A) ${ }^{+}$RNA was reverse transcribed using random hexamer primers (Pharmacia) and Superscript (BRL), and primers derived from the normal lip sequence were used to amplify $20 \mathrm{ng}$ of cDNA in $25 \mu \mathrm{l}$ of $p f u$ buffer (Stratagene) containing 10 pmoles each primer, 200 $\mu \mathrm{M}$ each dNTP and 0.5 units of $p f u$ DNA polymerase (Stratagene), with 30 cycles of $1 \mathrm{~min}$ at $92^{\circ}$, $1 \mathrm{~min}$ at $55^{\circ}$ and $3 \mathrm{~min}$ at $72^{\circ}$. PCR products, purified by gel electrophoresis and Geneclean (Bio101 Inc.), were digested with XhoI and XbaI before being cloned into XhoI/XbaI cut Bluescript $(\mathrm{SK}+)$. Inserts sequenced as already described. Primers pairs were as follows:
Primer 1: TACGCTCGAGACTTCTACCACAGCTTCCTG and

Primer 2: CGTACTCGAGACATCTTCCCCAGCCTGGAC Primer 3: GTCATCTAGAGCTATGTGACTGTACTCCAG and

Primer 4A: CATGTCTAGAACTCCCTGAACCTCCAGCTC.

\section{Results}

Isolation and sequencing of cDNA clones

The genomic clone MC15, isolated in our previous study, ${ }^{13}$ was used to screen an oligo-dT primed M426 human fibroblast library, ${ }^{17}$ and two clones of $3.0 \mathrm{~kb}$ (11A) and $2.6 \mathrm{~kb}(13 \mathrm{~A})$ were isolated. Complete bidirectional sequencing of clone $11 \mathrm{~A}$ generated a sequence of $3023 \mathrm{bp}$ including a 39-bp polyA tail. Although this clone corresponded in size to the $3.0 \mathrm{~kb}$ transcript detected by Northern analysis in a variety of human tumour cell lines (data not shown), the open reading frame present in this cDNA extended to the $5^{\prime}$ end of the sequence, raising the possibility that an additional $5^{\prime}$ sequence existed. The same probe was therefore used to screen 100000 clones from a randomly primed HT1080 cDNA library and three additional clones $12 \mathrm{C}, 5 \mathrm{~A}$ and $3 \mathrm{~B}$ were isolated. Of these, $3 \mathrm{~B}$ contained a $2.6-\mathrm{kb}$ insert, was primed at nucleotide 2542 with respect to $11 \mathrm{~A}$ and possessed an alternative $5^{\prime}$ end (Fig. 1(a)). Subsequent $5^{\prime}$ RACE analysis, using RNA derived from a human breast cell line, was used to demonstrate that $18 \mathrm{bp}$ of the $5^{\prime}$ sequence was missing from the original clone $11 \mathrm{~A}$. This upstream sequence, GAGGCTTCGGTTCCGGTG, did not, however, encode an upstream stop codon or an initiating methionine. In clone $11 \mathrm{~A}$, there are two methionines at the $5^{\prime}$ terminus which conform closely to the Kozak consensus sequence initiating at methionine, ${ }^{20}$ the first $7 \mathrm{bp}$ and the second 52 bp from the $5^{\prime}$ end of this clone. Neither is preceded by an in-frame stop codon. In clone $3 \mathrm{~B}$ the methionine at position 7 is absent (Fig. 2(a)). We have therefore chosen the methionine at position 52 as the most likely translation start site since this is present in both $11 \mathrm{~A}$ and $3 \mathrm{~B}$. If this is the case, lip encodes a single major open-reading frame of 2898 nucleotides predicting a protein of 966 amino acids. The sequence and the putative open-reading frame are shown in Fig. 2 (b).

\section{lip expression}

lip was expressed as a 3-kb transcript in all cell lines examined. These included the fibrosarcoma cell lines HT1080 and Hs913T, the leiomyosarcoma lines SKUT-1 and SK-LMS-1, the rhabdomyosarcoma cell lines A204, RMS and RD, the Ewings sarcoma cell line A673, the promyelocytic leukaemia cell line HL60 and the carcinoma cell line A431. In addition, lip was shown to be expressed in a variety of tissues including tonsil, spleen, renal cortex, lung, prostate, endometrium and breast (data not shown). 


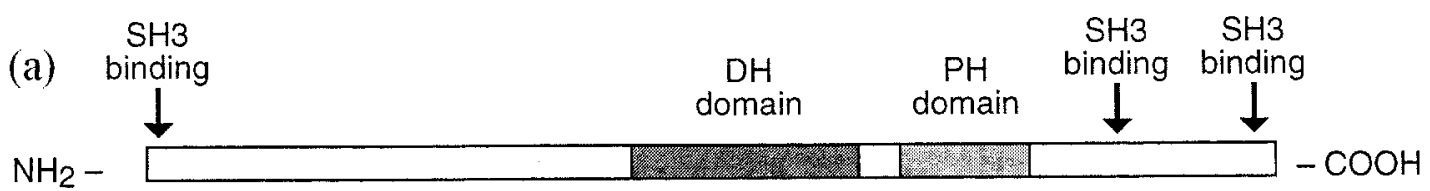

$5^{\prime}$

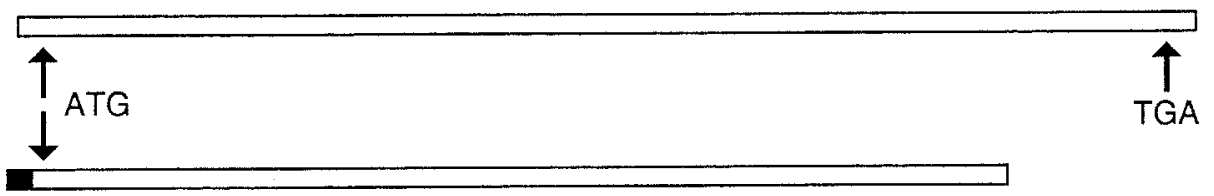

$11 \mathrm{~A}$

$3 B$

$500 \mathrm{bp}$

(b)

\begin{tabular}{cccc}
\hline 1 & 1 & 1 \\
1500 & 2000 & 2500 & $3000 \mathrm{bp}$
\end{tabular}
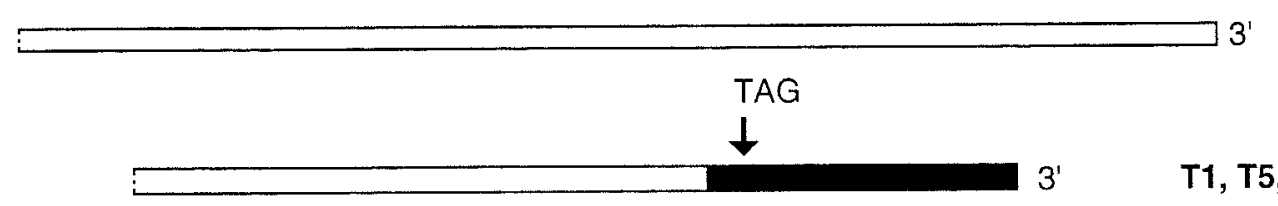

$\mathrm{T1}, \mathrm{T} 5, \mathrm{~T} 6, \mathrm{~T} 9, \mathrm{~T} 10$

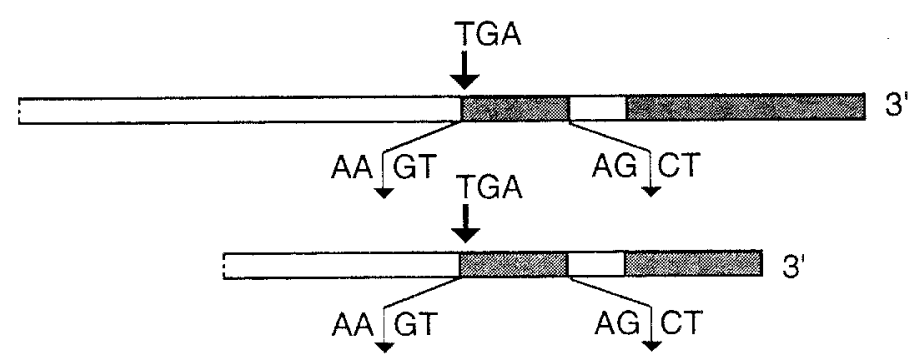

T3

$\mathrm{T} 2, \mathrm{~T} 7$

Fig. 1. (a) Schematic representation of the predicted lip protein, the cDNA clones $11 A$ and $3 B$. The Kozak methionine codon, ATG, present in both clones is marked, as is the in and frame stop codon. The alternative $5^{\prime}$ sequence in clone $3 B$ is demonstrated. The dbl homology $(D H)$ and pleckstrin homology $(P H)$ domains and the potential SH3 domain-binding sites are also shown. (b) Schematic representation of the $3^{\prime}$ ends of clone $11 \mathrm{~A}$, transfectant clones $T 1, T 5, T 6, T 9, T 10$, and T3, T2 and T7. Clones are aligned with respect to clone $11 \mathrm{~A}$. The alternative $3^{\prime}$ sequences in each of the three groups of transfectant clones are demonstrated by shading. The sequence at the junction sites between lip, non-lip sequences in transfectant clones T2, T3, T7 is shown and indicates that the 138-bp sequence inserted between lip nucleotides 2300 and 2301 may be a retained intron.

The predicted lip protein contains nucleotide exchange factor and PH domains

The predicted lip protein contains regions of moderate similarity to the previously described $d b l$ homology (DH) domain seen in the transforming oncogenes $d b l, v a v$, ect-2 and tim $;^{21}$ the yeast cell cycle gene $c d c 24$, the $b c r$ gene $\mathrm{e}^{22}$ and a nucleotide exchange factor for ras, p140-RasGRF. ${ }^{23}$ Using the FASTA search programme, the core region of this DH domain (amino acids 416-610) was most closely related to the $l b c, v a v, e c t-2$ and $c d c 24$ genes, demonstrating $33.8 \%, 25.9 \%, 25.5 \%$ and $24.3 \%$ identity respectively (Fig. 3(a)).

A second region of similarity to the pleckstrin homology $(\mathrm{PH})$ domain $^{24}$ has been found to span amino acids 649 to 758 . This domain, first identified as an internal repeat in pleckstrin, the major substrate of protein kinase $\mathrm{C}$ in platelets, ${ }^{25}$ has been identified in a number of other proteins including the products of the vav, dbl, rasgrf, bcr, $l b c$ and $c d c 24$ genes. lip shows $24 \%$ identity with the pleckstrin C-terminal $\mathrm{PH}$ domain. Although the identity is low, the family members noted so far have exhibited only $21-25 \%$ identity. All five of the previously defined subdomains can be identified, and the most highly conserved residues which define these subdomains ${ }^{25}$ are also conserved in lip (Fig. 3(b)).

The C-terminal 200 amino acids are relatively proline rich $(16 \%)$ and contain a number of sites which conform to the minimal consensus sequence for SH3 domain binding sites, $\mathrm{P}-\star / \mathrm{p}-\mathrm{X}-\mathrm{P}$ $(\mathrm{P}=$ proline,$\quad \star / \mathrm{p}=$ usually hydrophobic/proline, $\mathrm{X}=$ not conserved). ${ }^{26}$ There is an additional potential SH3 domain-binding sequence in the amino 
(a)

(b)

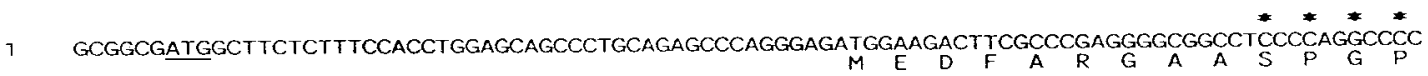

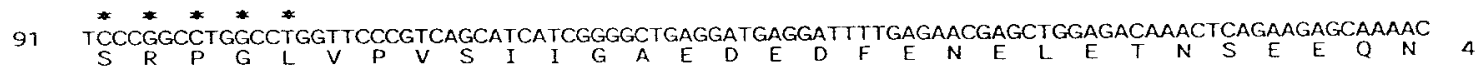

181 AGCCAGTTCCAGAGCCTGGAGCAGGTGAaGCGGCGCCCAGCCCACCTCATGGCCCTCCTGCAGCACGTGGCCCTGCAGT TTGAGCCAGGA

271 CCCCTGCTTTGCTGTCTGCATGCCGACATGCTGGGCTCACTGGGCCCCAAGGAGGCCAAGAAGGCCTTCCTGGACTTCTACCACAGCTIC

361 CTGGAGAAGACAGCGGTTCTCCGGGTGCCGGTCCCTCCCAACGTCGCCTTTGAACTTGACCGCACTAGGGCTGACCTCATCTCCGAGGAT 451 GTCCAGCGGCGGTTCGTGCAGGAGGTGGTGCAAAGCCAGCAGGTAGCCGTGGGCCGGCAGCTGGAGGACTTCCGTTCCAAGCGGCTCATG

541 GGCATGACGCCCTGGGAGCAGGAGCTGGCCCAGCTGGAGGCTTGGGTTGGGCGGGACCGAGCCAGCTACGAGGCCCGGGAGCGGCACGTG M T W E R E A

631 GCGGAGCGGCTGCTCATGCACCTGGAGGAGATGCAACATACCATCTCTACCGACGAAGAAAAGAGTGCTGCCGTGGTCAACGCCATTGGC

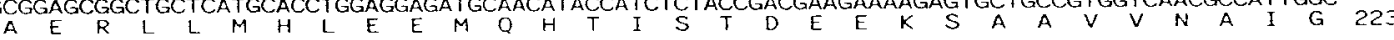

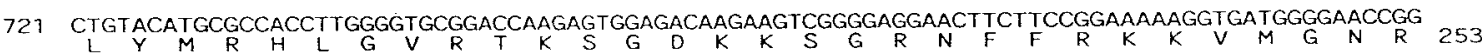
811 CGGTCGGACGAGCCTGCCAAGACCAAGAAGGGGCTGAGCAGCATCCTGGATGCCGCCCGCTGGAACCGGGGAGAGCCCCAGGTTCCAGAT R S F P A K K K G L S S I L D A A R W N R G E P Q V P D 283

901 TTTCGACACCTCAAAGCAGAGGTTGATGCCGAGAAGCCAGGTGCTACAGACCGGAAGGGAGGCGTGGGGATGCCCTCTCGGGACCGGAAT

991 ATCGGGGCTCCTGGGCAGGACACCCCTGGAGTCTCTCTGCACCCTCTGTCCCTGGACAGCCCAGACCGGGAACCAGGTGCTGACGCCCCC 1081 CTGGAGCTGGGGGACTCATCCCCGCAGGGCCCAATGAGCCTGGAGTCCTTGGCGCCCCCAGAGAGTACCGACGAGGGGGCCGAAACCGAG

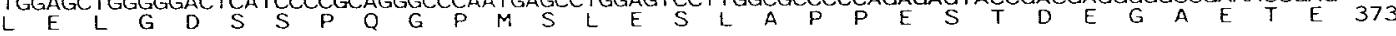

1171 AGCCCCGAGCCTGGAGATGAGGGGGAGCCGGGGCGGTCGGGACTGGAGCT TGAACCAGAAGAGCCTCCCGGCTGGCGGGAACTCGTCCCC

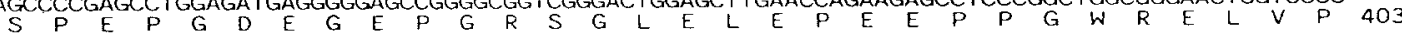
1267: CCAGACACCCTGCACAGCCTGCCCAAGAGCCAGGTGAAGCGGCAGGAGGTCATCAGCGAGCTGCTGGTGACAGAGGCGGCCCACGTGGCC $\mathrm{V}$ i

1351 ATGCTGCGGGTGCTGCACGACCTCTTCT TCCAGCCCATGGCAGAATGCCTGTTCTTCCCCTTGGAGGAGCTGCAGAACATCTTCCCCAGC

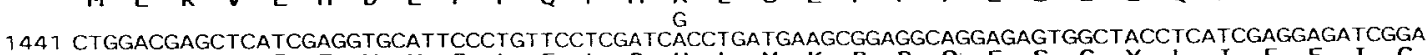
L D E L I E V H S L F L D H L M K R R Q E S G Y L I E E I G 1531 GACGTGCTGCTGGCCCGGTTTGATGGTGCTGAGGGCTCCTGGTTCCAGAAAATCTCCTCCCGCTTCTGCAGCCGCCAGTCATTTGCCTTA 1621 GAGCAGCTCAAAGCCAAGCAACGCAAGGACCCTCGGTTCTGTGCCTTCGTGCAGGAAGCTGAGAGCCGCCCGCGGTGCCGCCGCCTGCAG

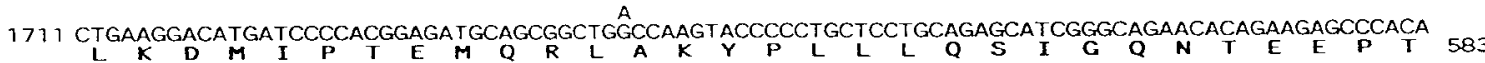
1801 GAACGGGAGAAAGTGGAGCTGGCAGCCGAGTGCTGCCGGGAAATICTACACCACGTCAACCAAGCCGTGCGTGACATGGAGGACCTGCTG E R E K V E L A A E C C R E I L H H V N Q A V R D M E D L L G13

1897 AGGCTCAAGGACTATCAGCGGCGCCTGGACTTGTCCCACCTTCGGCAGAGCAGCGACCCTATGCTGAGCGAGTTCAAGAACCTGGACATC 1981 ACCAAGAAGAAATTGGTCCACGAGGGCCCACTGACGTGGCGGGTGACTAAGGACAAGGCAGTGGAGGTGCATGTGCTGCTGCTGGACGAC 673

Fig. 2. (a) The alternative $5^{\prime}$ sequence from clone $3 B$ and (b) the nucleotide and predicted amino acid sequence of the lip cDNA clone $11 \mathrm{~A} .5^{\prime} \mathrm{RACE}$ analysis has demonstrated the presence of an additional 18-bp 5' sequence, GAGGCTTCGGTTCCGGTG, which is missing from clone $11 \mathrm{~A}$. The amino acid sequence commences at the second Kozak methionine at nucleotide position 52 . The first methionine at position 7 (absent from clone $3 B$ ) is underlined, the divergent sequence in clone $3 B$ is overlined. The amino acid substitutions within the DBL homology $(D H)$ domain, introduced by the mismatches between clone $11 \mathrm{~A}$ and the transfectant clones, are shown above the nucleotide sequence, $A \longrightarrow G$ at nucleotide 1481, G $\rightarrow$ A at nucleotide 1747. The DH domain is shown in bold. The PH domain is flanked by arrowheads, potential SH3 domain-binding sites are marked by asterisks above the sequence. The positions at which 11 A sequence and the sequence in clones T1, T3 diverge are marked with arrows. The stop codon TGA is marked with an asterisk below the sequence. Nucleotide sequence is numbered on the left and amino acid sequence on the right. (c) Nucleotide and predicted amino acid sequence of the $3^{\prime}$ end of transfectant cDNA clone T1. The nucleotide and predicted amino acid sequences are numbered with respect to clone $11 \mathrm{~A}$, and sequence common to both clones $11 \mathrm{~A}$ and $T 1$ is underlined. An arrowhead marks the site of the rearrangement which replaces 188 amino acids from the predicted lip protein with 15 novel residues. The in-frame stop codon is marked with an asterisk. (d) Nucleotide and predicted amino acid sequence of the $3^{\prime}$ end of transfectant clone T3. Nucleotide and amino acid sequences are numbered with respect to clone $11 \mathrm{~A}$, and sequences common to both clone $11 \mathrm{~A}$ and T3 are marked with arrowheads and underlined. The in-frame stop codon is marked with an asterisk. The rearrangement in clone T3 replaces 217 amino acids at the $3^{\prime}$ end of clone $11 \mathrm{~A}$ with a single novel residue. 
2071 CTGCTGCTGCTGCTCCAGCGCCAGGACGAGCGGCTGCTGCTCAAGTCCCATAGCCGGACACTGACGCCCACGCCCGATGGCÄAGACCATG

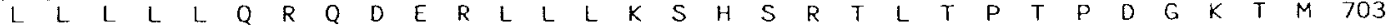

2161 CTGCGGCCCGTGCTGCGGCTCACCTCCGCCATGACCCGCGAGGTGGCCACCGATCACAAAGCCTTCTACGTCCTITTTACCTGGGACCAG

$L R P P L R$ L T S A M T R E V A T D H K A F Y V L F T W D Q 733

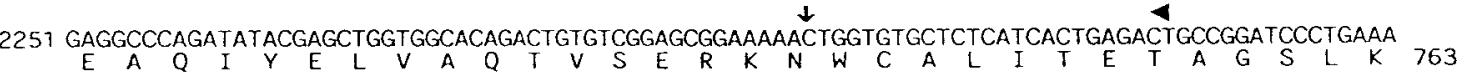
$\downarrow$

2341 GTCCCTGCCCCTGCCTCTCGCCCTAAGCCCCGGCCCAGCCCGAGCAGCACCCGAGAACCCCTCCTCAGCAGCTCTGAGAACGGCAATGGT

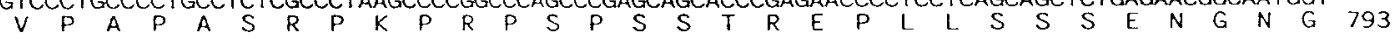

2431 GGCCGAGAGACGTCTCCAGCTGATGCCCGGACCGAGAGAATCCTCAGTGACCTCCTGCCCTICTGCAGACCAGGCCCCGAGGGCCAGCTC

$G R$ E T S P A D A R T E R I L S D L L P F C R P

* * * ***** * *

2521 GCTGCCACGGCCCTTCGGAAAGGAGTTGGAGGAGGAATTTTGCCGCCTGAGACCCCTCCTGTCTCAGCTTGGGGGGACTCTGTCCCCCA

A A T A L R K G V G G G I L P P E T P P V S A W G E L C P P 853

2611 GCCTGGCTGCACTTGAGGTTCCCGCCCAGGAAGGCCTTTTGCAAGAAGGAGAGGAATGGGGGAGAGGACGTGAGGGACCACCCCCACCCA A W L H L R F P P R K A F C K K E R N G G E D V R D H P H P 883

$* * * * *$

2701 CACAGCTGCCGCAGCATCTCACACCCCGAGGGCCTGAGGAGAGGGAGCTGTGGGCCACGCCTGGGAGGGGCCCAGCTGGGGTTACTGGCC

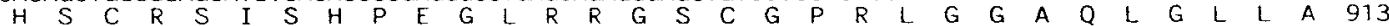

****** * *

2791 CCGCATGAGCCTCGGCCATCTCTCCCTCCTGCCCTCTGCTTGGGGGACTCAGGGCTCCATTCTGGAGGGCACCACGGTGACCCGGGCCAT

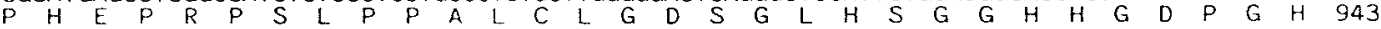
* ******** * * * *

2881 CTCAGTATTGCCTGTGGGGGCCACCCCTCCACCCCCACCCCCAAGTGCCITCGCTCTGTTTTTATACCCTGAATTGGAGGTTTATITTTT

$L$ S I A C G G H P S T P T P K C L R S V F I P *

2971 AATATATATTATCTAAAAAAAAAAAAAAAAAAAAAAAAAAAAAAAAAAAAAAA

(c)

2251 GAGGCCCAGATATACGAGCTGGTGGCACAGACTGTGTCGGAGCGGAAAAACTGGTGTGCTCTCATCACTGAGACTGCCGGATCOCTGAAA

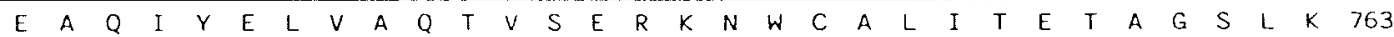

2341 GTCCCTGCCCCTGCCTCTCGCCCTAAGCCCCGGCCCAGCCCGAGCAGAGACAGGGTCTCACTCTGTTGCCCAGCCTGGAGTACAGTCACA

$\begin{array}{lllllllllllllllllllllllllllllllll}V & P & A & P & A & S & R & P & K & P & R & P & S & P & S & R & D & R & V & S & L & C & C & P & A & W & S & T & V & T & 793\end{array}$

2431 TAGCTCACTACAGCCTCAAATTCCTGAGCTTAAGTGATCCTTCAGCCCCAGCCTCCCAAGTAGCTGGAACTATAAGCTAGCATCATCACA

2521 CCTGGCAAATTTITAAAAATTTTTITTATAGACACTGTCTCACTATGTTGCCCTGGCTGGTCTTGAACTCCTGACCTCAAGCTATCCTCC

2611 TGCCTCAGCCTCTCAAAACTCTGGGATTACAGGCATGAGCCAACGCACTTAGCCAAAAAAATTTTAATTATAAAATATATCATTTTGGTT

2701. CCTAACATTTTGTATGTAAGCACCCTITITAAAAACAACAATAAAATCCTATGGACCTCCAAAAAAAAAAAAAAAAA

(d)

2251 GAGGCCCAGATATACGAGCTGGTGGCACAGACTGTGTCGGAGCGGAAAAAGTGAGGGGGGGTCTGAGTTCTGAGTGTGGGTGGGGACGC

E $A \begin{array}{llllllllllllllllllllllllllll} & \text { A } & I & Y & E & L & V & A & Q & T & V & S & E & R & K & K & *\end{array}$

2341 CCAGGTTTCTGGGTTCCCAGGGACAGGAGGGCTGTGGGGAGGCCCTGGCATAGGGTCTGGGGGCTCTGACTGCCCAGGGATTTGGCCTTT

2431 CTCCCCAGCTGGTGTGCTCTCATCACTGAGACTGCCGGATCOCTGAAAGTCCCTGCCCCTGCCTCTCGCCCTAAGCCCCGGCCCAGCCCG

2521 AGCAGGTGAGGGGGGCCATGGAGAGAGCTGGAGGTTCAGGGAGTGGGGCCGGAAGGCGGGGCAGGCTTCCCTCCACAACTCCAGAACCGT

2611 CTCTGTGTGAGCATGCACATGTGTGAGGGCAACATGGCAGGAACCTGTCTCTACTAAAAATACAAAAAAATTATTGGGCATAGTGGTGCG

2701 TGCCTGTGGTCCCAGCTGCITGGGAGGCTGAGGAGGGAGGATCAATTAAGCCCAGCGTGTGAAGTTGCAGTGACCAGTCTGGGTGATAAA

2791 GCAAGACTCTGTCGCAAAAAAAAAAAAAAAAAA

Fig. 2. Continued.

terminal region. AASPGPSRPGL, which closely resembles the $\mathrm{SH} 3$ domain binding sequences seen in the human dynamin protein, a microtubule binding protein which has GTPase activity and is thought to be involved in vesicle trafficking. ${ }^{27} \mathrm{~A}$ Prosite database motif search reveals 13 consensus sequences for protein kinase C phosphoryation, 13 consensus sequences for casein kinase phosphorylation, a single consensus sequence for tyrosine phos- phorylation at residue 487 and a single potential site for mitogen-activated protein kinase (MAPK) phosphorylation, $\mathrm{P}-\mathrm{X}-\mathrm{S} / \mathrm{T}-\mathrm{P}$, at residue 954 (Fig. 2(b)).

LIP shares identity with p115-RHOGEF, an exchange factor for $R H O A$

p115-RHOGEF was identified using RHOA as an affinity-purified ligand, and subsequently cloned 
(a)

\begin{tabular}{|c|c|c|}
\hline IP & 13 & 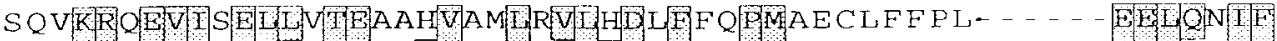 \\
\hline $\mathrm{ECT} 2$ & 77 & 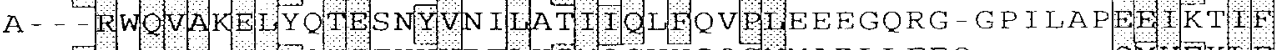 \\
\hline $\mathrm{BC}$ & 71 & 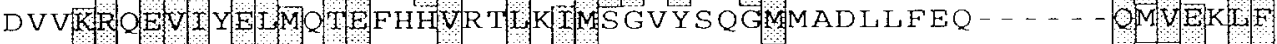 \\
\hline IM & 93 & 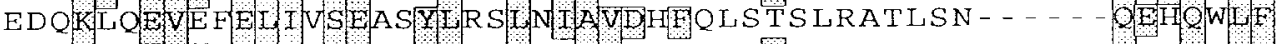 \\
\hline DBL & 496 & 1YTULLGYRAEMDNPEMFDLMPPL \\
\hline VAV & 91 & CCCHRE QQME EKYTDTMGS TQYH \\
\hline $\mathrm{CDC} 24$ & 160 & $291 \mathrm{LDSNLIT}$ - - \\
\hline GRF & 237 & 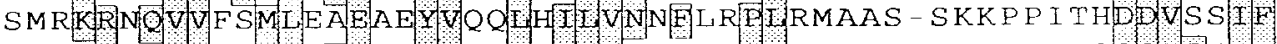 \\
\hline $\mathrm{BCR}$ & 495 & 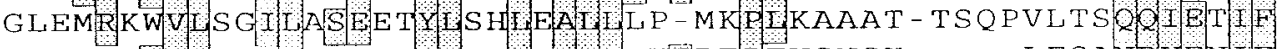 \\
\hline OS & 97 & dYIREINUIJ KVEREGFVSNSK- - - - LFS \\
\hline
\end{tabular}

GYLIEEIGULAARFDGAEGSWFQKTSS

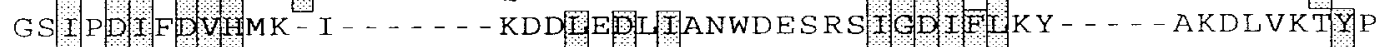

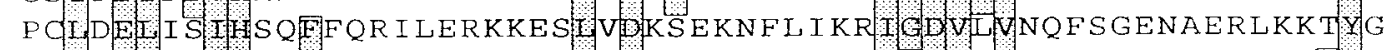

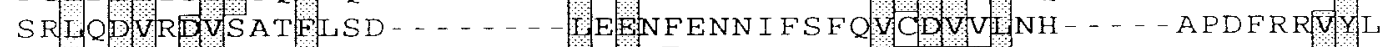

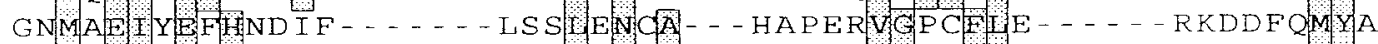

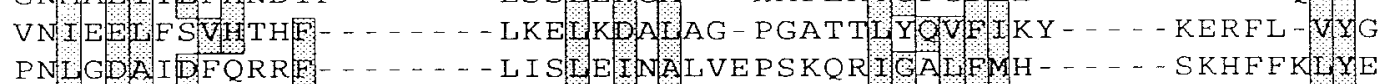
GRF $B C R$ SOS

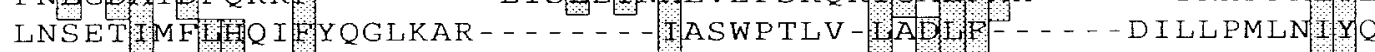

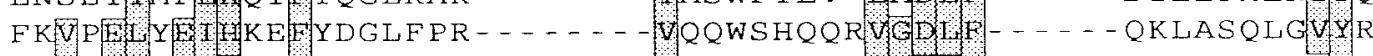

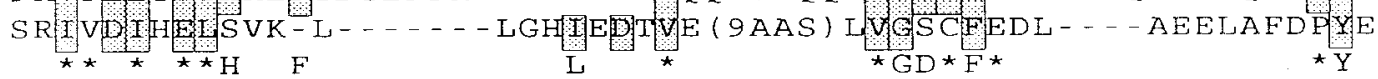

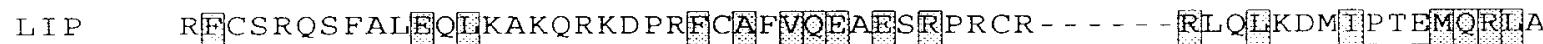

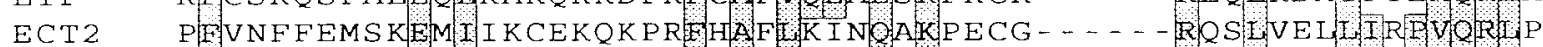
LBC KIFCGQHNQSVNYFKDLYAKDKRFQAFUKKKMSSSVVR - . - -RLGIPECIILVTPRIT

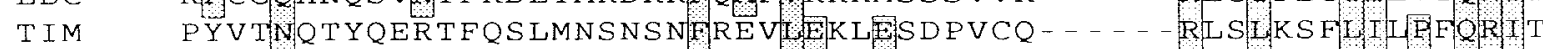

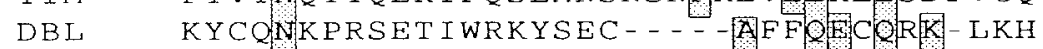
VAV RYCSQVESASKHTDQVATAREDVOMKHEACSQRANNG CDC24 PWSIGQNAA IEETISSTLHK- - - MRVIESGRF I INN -

GRF

$\mathrm{BCR}$ SOS EFVRNHQYSIJIIAHC - KQNRDEDKLHKOYGAKPDCEER THETFITTYMMEQTP AFVDNYGVAMEMAEKCCQANAQ AAE I SENLRARSS NDAKD PTTRNSIETLLYKPVDRUT SYARDILRPGFHDRFLSQLSKPGAALYLGS IGEGFKEAV - - - QYVLPRLIIIAPUYHCL RLRIDS YHTFEYORIT

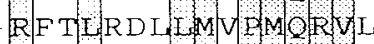

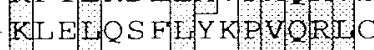
$R \underset{\mathrm{L}}{\mathrm{L} *} \mathrm{P} * \mathrm{QR}$ *

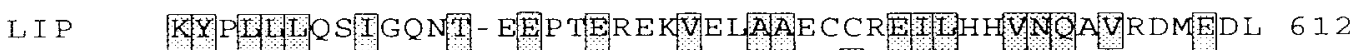
ECT2 SVATHNTHKKHTADENPDKSTHEKAJGSTKEMMTHINEDKRKTEAQ 473

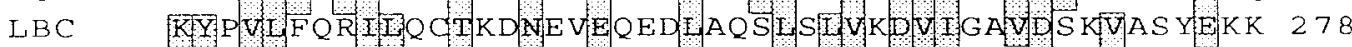
TIM RLKIUINIHKRTQPGSSEEAEAT KAHHATEQTIRD CNNNMQSMR - 287

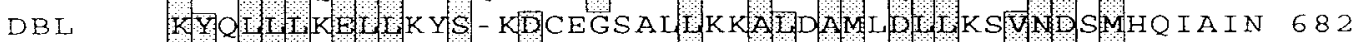

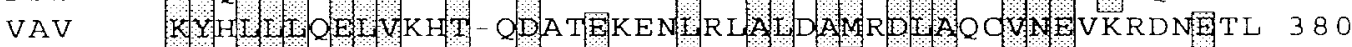

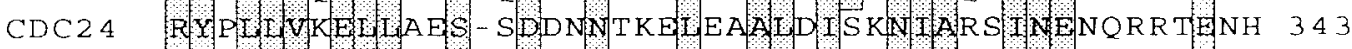

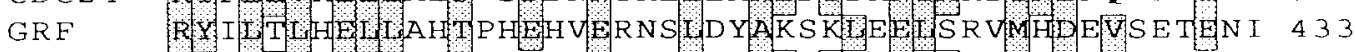

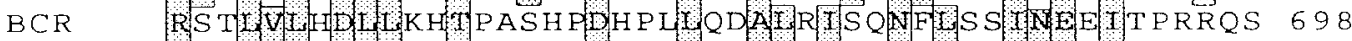

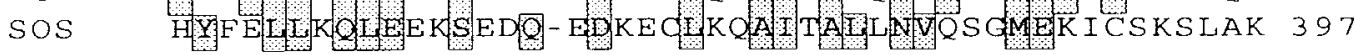

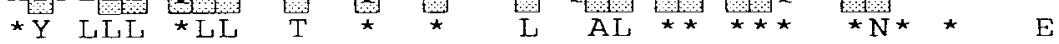

Fig. 3. (a) Computer-generated alignment of the DH domain in LIP, ECT-2, LBC, TIM, DBL, VAV, CDC24, p140RASGRF, $B C R$ and murine SOS using the Clustal V programme. The consensus sequence is indicated below. A capital letter indicates six or more of the proteins possess an identical amino acid at this position, and an asterisk that the amino acid is conserved in six or more of the proteins. Amino acids are numbered on the left. Sequences were obtained from the GENBANK database via Northwick Park Hospital, Middlesex, UK. (b) Alignment of pleckstrin N, pleckstrin C with LIP, VAV,DBL, CDC24, ECT-2, p14ORASGRF, BCR and SOS, over the PH domain. The proteins were aligned as previously described. ${ }^{30}$ The conserved amino acid residues which anchor the five previously described subdomains ${ }^{24}$ are shown in capitals below the alignment, with additional conserved residues marked with an asterisk.

from human fetal brain cDNA libraries. ${ }^{14}$. Comparison of the cDNA sequences obtained for lip and p115-RhoGEF shows alternative $5^{\prime}$ untranslated sequences, sequence variations resulting in six isolated amino acid substitutions in the predicted protein sequence and an alternative $3^{\prime}$ end. 
(b)

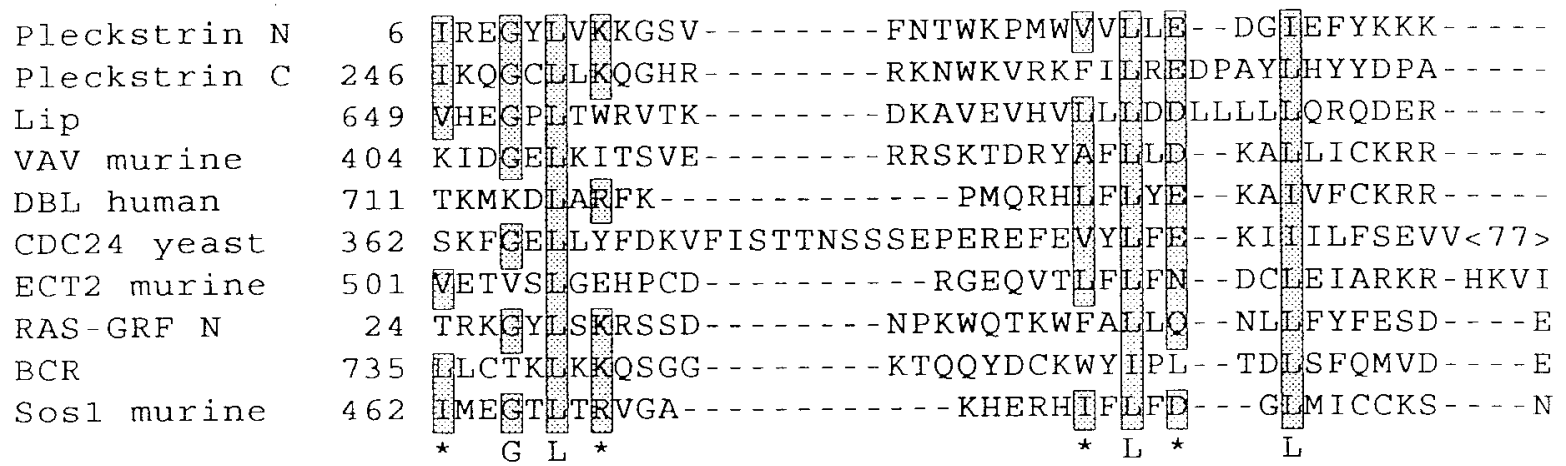

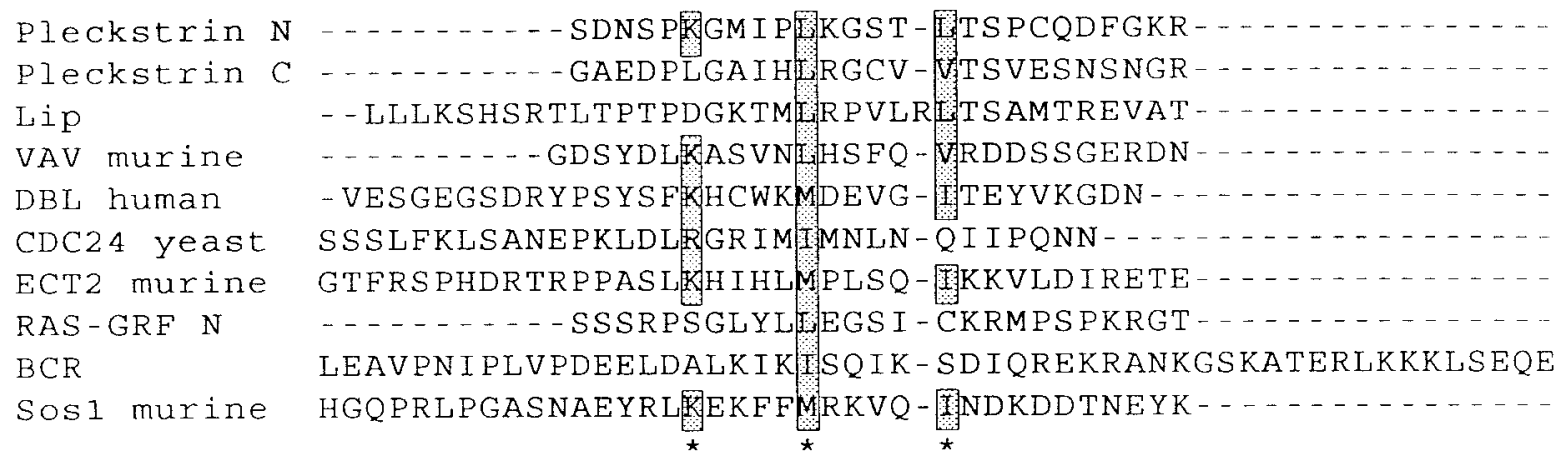

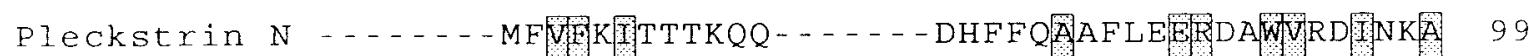

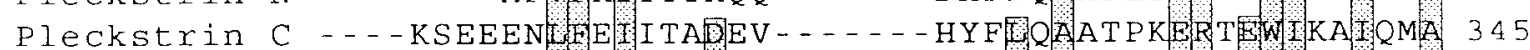
Lip VAV murine DBL human CDC2 4 yeast ECT2 murine RAS-GRF N $B C R$

Sos 1 murine

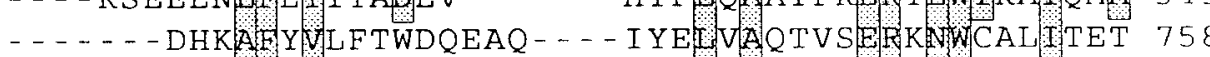

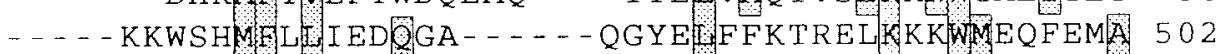

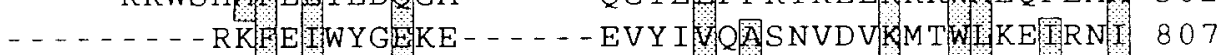

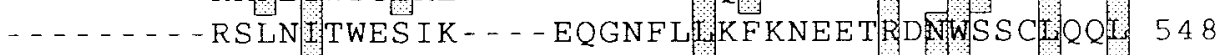
- - - - DCHNATAHLVRPPTEQ - ANVLLS FQMTSEELPKESWTKMLCRH 615

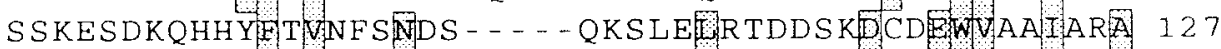

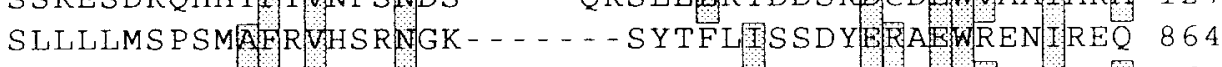

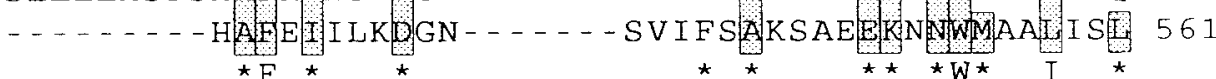

Fig. 3. Continued.

The 5' sequence identified for $\mathrm{p} 115-\mathrm{RhoEF}$ is a shortened version of that identified in clone $3 \mathrm{~B}$ derived from an HT1080 cDNA library, and clone $3 \mathrm{~B}$ extends this sequence by $26 \mathrm{bp}$. The alternative $5^{\prime}$ sequence identified in clone $11 \mathrm{~A}$ and with $5^{\prime}$ RACE analysis using RNA from a human breast cell line contains an in-frame upstream methionine and may represent an alternative start site for translation initiation (Fig. 2b)

With the exception of the six amino acid substitutions, $\mathrm{D} \rightarrow \mathrm{E}$ (codon 257), $\mathrm{P} \rightarrow \mathrm{A}(\operatorname{codon} 259), \mathrm{R} \rightarrow \mathrm{A}$ $(\operatorname{codon} 433), \mathrm{R} \rightarrow \mathrm{H}(\operatorname{codon} 477), \mathrm{T} \rightarrow \mathrm{A}(\operatorname{codon} 566)$ and $\mathrm{R} \rightarrow \mathrm{S}$ (codon 776), lip and p115-RhoGEF are identical until amino acid 830. At this point the sequence AAA/GGAGTT in lip is replaced by AAA/ GTGCTG in p115-RhoGEF. The sequence in p 115-RhoGEF is a potential intron splice donor site, and the novel sequences seen in lip may have arisen as a result of alternative splicing.

Analysis of the lip gene in the primary liposarcoma and in transfectant cell lines

Hybridization of clone $11 \mathrm{~A}$ to Southern blots of EcoRI-digested normal genomic DNA gave bands of 15 and $7 \mathrm{~kb}$. These bands were present in tumour DNA from the original liposarcoma, but bands of 17 and $3.7 \mathrm{~kb}$ were observed using DNA derived from primary and secondary transfectant cell lines (data not shown). This data indicated that the lip gene might be activated by rearrangement, but if this were the case then the rearrangement had taken place during the transfection process and was not present in the primary liposarcoma. To further de- 
lineate any role lip gene rearrangement might play in sarcoma development, an additional 52 primary tumours were evaluated by Southern analysis of EcoRI-digested tumour DNA. This group included 12 malignant fibrous histiocytomas, 10 leiomyosarcomas, nine liposarcomas, five malignant peripheral nerve sheath tumours, four rhabdomyosarcomas, two synovial sarcormas, one chondrosarcoma, one fibrosarcoma, one post-irradiation spindle cell sarcoma, one dermatofibrosarcoma protuberans, one fibromatosis, two haemangiomas, one lipoma, one neurilemmoma and one clear cell carcinoma. In no case was any rearrangement detected.

\section{Analysis of the mechanism of lip activation}

To examine the mechanism of activation of lip, we prepared an oligo-dT primed cDNA library using RNA from the lip transfectant cell line. Screening 200000 clones using clone $11 \mathrm{~A}$ as a probe resulted in the identification of 10 partial length cDNA clones ( $\mathrm{T} 1$ to $\mathrm{T} 10$ ) that were characterized by DNA sequencing. Clone T4 exhibited $88 \%$ DNA sequence identity and $98 \%$ protein sequence identity to the human lip sequence, and probably corresponds to a mouse lip clone. Analysis of the remaining clones revealed that they could be divided into three groups, each of which exhibited loss of the $3^{\prime}$ lip sequences. In clones $\mathrm{T} 1,5,6,9$ and 10, lip was replaced $3^{\prime}$ to nucleotide 2387 by a new sequence that in database searches showed regions of homology to human alu repeat sequences. This rearrangement replaces 188 amino acids at the carboxy terminus of the predicted lip protein with 15 novel amino acids (Figs 1 (b) and 2(c)). This result was consistent with Southern analysis data showing that probes prepared from $3^{\prime}$ fragments of the normal lip cDNA failed to detect human sequences in lip primary and secondary transfectants (results not shown). At position 1747 there was a $\mathrm{G} \longrightarrow \mathrm{A}$ base change converting an alanine in lip to a threonine in the transfectant lip clone at codon 566. In addition, T5 which was the longest of all the clones, showed an $\mathrm{A} \longrightarrow \mathrm{G}$ base change at position 1481 substituting an arginine in lip for a histidine in the activated lip clone at codon 477 . The presence of these alterations, which lie in the $\mathrm{DH}$ domain, was confirmed by sequencing a PCR product generated from reverse-transcribed lip transfectant RNA, using primers flanking this region.

The second group of cDNAs was represented by a single clone, $\mathrm{T} 3$, which also encoded the $\mathrm{G} \rightarrow \mathrm{A}$ alteration at codon 1747 . In $\mathrm{T} 3$, lip sequence is interrupted immediately after nucleotide 2300 by $138 \mathrm{bp}$ of novel sequence, followed by an 87-bp sequence corresponding to nucleotides 2301-2387 of lip and then by $280 \mathrm{bp}$ of additional novel sequence and a polyA tail. The novel sequences in T3 were unrelated to those in the first group of clones discussed above. In T3, lip is truncated by 216 amino acids as a stop codon is introduced immediately following the break-point (Figs 1(b) and 2(d)). This alteration removes an invariant tryptophan from the $\mathrm{PH}$ domain discussed earlier. Analysis of the junctions between lip sequences and novel sequences in clone $\mathrm{T} 3$ suggests that the $138 \mathrm{bp}$ of the novel sequence inserted between nucleotides 2300 and 2301 may be a retained intron (Fig. 1(b)).

The third group of cDNA clones represented by clones T2 and T7 were identical to clone T3 except that their $3^{\prime}$ ends were respectively 133 and $136 \mathrm{bp}$ upstream from the start of the polyA tail in T3.

\section{Discussion}

Here, we describe the cloning and characterization of a gene detected in NIH3T3 transfection experiments using DNA from a human liposarcoma. This gene, lip, shares homology with the oncogenes $d b l$, $v a v, e c t-2$, tim and $l b c$, and the yeast cell cycle gene cdc24, encompassing both the $\mathrm{DH}$ and the $\mathrm{PH}$ domains. CDC24 functions as an exchange factor for the RHO-like protein CDC42 in budding yeast, ${ }^{27}$ $\mathrm{DBL}$ is known to act as an exchange factor for CDC42Hs and RHOA, ${ }^{28}$ ECT-2 binds RHOC and RAC $1^{11}$ and LBC acts as an exchange factor for RHOA, RHOB and RHOC. ${ }^{29}$ Moreover, with the exception of six isolated amino acid differences, lip is identical over its $\mathrm{N}$-terminal 830 amino acids (including $\mathrm{DH}$ and $\mathrm{PH}$ domains) to the recently identified protein $\mathrm{p} 115 \mathrm{RhoGEF}$, which functions as an exchange factor for RHOA. ${ }^{14}$ Guanine nucleotide exchange factors bind preferentially to the nucleotide-depleted state of G-proteins, and by stimulating the release of GDP they promote the subsequent binding of GTP, and hence G-protein activation. The $\mathrm{DH}$ domain appears to function primarily as an GDP-GTP exchange domain for members of the RHO family. The $\mathrm{PH}$ domain ${ }^{30}$ appears to be involved in a wide variety of molecular interactions. The C-terminal regions of several $\mathrm{PH}$ domains bind to the beta-gamma subunits of heterotrimeric Gproteins, ${ }^{31,32}$ and the $\mathrm{N}$-terminal region to phosphoinositol-4,5-bisphosphate, ${ }^{33}$ which implies that the $\mathrm{PH}$ domain is important for membrane localization. In all the exchange factors which possess both domains, the PH domain is located immediately C-terminal to the $\mathrm{DH}$ domain. This suggests that the $\mathrm{PH}$ domain is important for the function of the exchange factor domain.

In transfectants, lip appears to be activated by C-terminal truncation, a rearrangement which appears to have taken place during the transfection procedure, and was not present in the primary liposarcoma. Moreover, examination of an additional 52 tumours by Southern analysis revealed no evidence in support of a role for lip rearrangement in sarcoma development.

Support for C-terminal truncation as the mechanism of lip activation comes from evidence that 
pll5RhoGEF is activated for transformation in the NIH3T3 focus-forming assay by both N- and C-terminal truncation. ${ }^{14}$ Moreover, additional members of this protein family have also been shown to be activated by truncation. ${ }^{8,11,34-36}$ In general, these activating mutations appear to involve the removal of putative regulatory C-terminal and/or N-terminal domains, but leave the $\mathrm{DH}$ and $\mathrm{PH}$ domains intact. $^{37}$ In the oncogene $l f c$, mutation of the conserved tryptophan residue in the PH domain abolishes transforming activity as does removal of only three amino acid residues from the $\mathrm{N}$-terminal region of its $\mathrm{DH}$ domain. Deletions which do not involve the $\mathrm{DH}$ or $\mathrm{PH}$ domains do not abolish transforming activity. In addition, replacement of the PH domain with an alternative membrane-localizing signal such as an isoprenylation or myristoylation site restores transforming activity. ${ }^{37}$ This supports the view that the $\mathrm{PH}$ domain is required for DH domain activity, which appears to be promoted by localizing the exchange factor to the plasma membrane.

lip is predicted to encode a protein with a number of differences from $\mathrm{p} 115 \mathrm{RhoGEF}$. In addition to differences in six amino acid residues within the N-terminal 830 amino acids. the C-terminal 133 amino acids are unique to lip. Within this $\mathrm{C}$-terminal region there are a number of proline-rich regions which conform to the minimal consensus sequence for $\mathrm{SH} 3$ domain binding sites. ${ }^{26}$ SH3 domain-binding sites have been identified in a number of proteins including, for example, the mammalian RAS exchange factor mSOS. ${ }^{38}$ In SOS, these proline-rich sequences mediate binding to the $\mathrm{SH} 3$ domains of the adaptor protein GRB2, ${ }^{39,40}$ an interaction which recruits SOS to the cell membrane, where it is pivotal in the signal transduction pathway from receptor tyrosine kinases to RAS proteins. LIP and p115RhoGEF may represent alternatively spliced forms of the same gene, and if the proline-rich sequences present in LIP serve a regulatory function, perhaps via a role as $\mathrm{SH} 3$ domain-binding sites, then this alternative splicing may represent a mechanism by which LIP/ p115RHOGEF function is regulated.

\section{Acknowledgements}

The M426 fibroblast cDNA library was kindly provided by Dr S. Aaronson. This work is supported by grants from the Cancer Research Campaign and the Medical Research Council.

\section{References}

1 Cooper CS, Park M, Blair DG, et al. Molecular cloning of a new transforming gene from a chemicallytransformed human cell line. Nature $1984 ; 311: 29-33$.

2 Takahashi M, Ritz J, Cooper GM. Activation of a novel human transforming gene, ret, by DNA rearrangement. Cell 1985; 42:581-8.
3 Martin-Zanca D, Hughes SH, Barbacid M. A human oncogene formed by the fusion of truncated tropomyosin and tyrosine kinase sequences. Nature 1986; 319:743-8.

4 Young D, Waitches G, Birchmeier, et al. C. Isolation and characterization of a new cellular oncogene encoding a protein with multiple potential transmembrane domains.Cell 1986; 45:711-9.

5 Eva A, Aaronson SA. Isolation of a new human oncogene from a diffuse B-cell lymphoma. Nature 1985; 316:273-5.

6 Shimizu K, Nakatsu Y, Sekiguchi, et al. Molecular cloning of an activated human oncogene homologous to $v$-raf, from primary stomach cancer. Proc Natl Acad Sci USA 1985; 82:5641-5.

7 Taira M, Yoshida T, Miyagawa K, et al. cDNA sequence of human transforming gene hst and identification of the coding sequence required for transforming activity Proc Natl Acad Sci USA 1987; 84:2980-4.

8 Katzav S, Martin-Zanca D, Barbacid M. vav, a novel human oncogene derived from a locus ubiquitously expressed in haemopoietic cells. EMBO 1989; 8:2283-90.

9 Janssen JWG, Schulz AS, Steenvoorden ACM, et al. A novel putative tyrosine kinase receptor with oncogenic potential. Oncogene 1991; 6:2113-20.

10 O'Bryan JP, Frye RA, Cogswell PC, et al. axl, a transforming gene isolated from primary human myeloid leukaemia cells, encodes a novel receptor tyrosine kinase. Mol Cell Biol 1991; 11:5016-31.

11 Miki T, Smith CL, Long JE, et al. Oncogene ect-2 is related to regulators of small GTP-binding proteins. Nature 1993; 362:462-5.

12 Toksoz D, Williams DA. Novel human oncogene $l b c$ detected by transfection with distinct homology regions to signal transduction products. Oncogene 1994; 9:621-8.

13 Gill S, Stratton MR, Patterson H, et al. Detection of transforming genes by transfection of DNA from softtissue tumours. Oncogene 1991; 6:1651-6.

14 Hart MJ, Sharma S, elMasry N, et al. Identification of a novel guanine nucleotide exchange factor for the RHO GTPase. F Biol Chem 1996; 271:25452-8.

15 Wilkinson M. RNA isolation: a mini-prep method. Nucleic Acids Res 1988; 16:10933.

16 Mitchell PJ, Cooper CS. The human tpr gene encodes a protein of 2094 amino acids that has extensive coiled-coil regions and an acidic C-terminal domain. Oncogene 1992; 7:383-8.

17 Miki T, Matsui T, Heidaran MA, et al. An efficient directional cloning system to construct cDNA libraries containing full-length inserts at high frequency. Gene $1989 ; 83: 137-46$.

18 Chomczynski P, Sacchi N. Single-step method of RNA isolation by guanidinium thiocyanatephenol-chloroform extraction. Anal Biochem 1987; 162:156-9.

19 Sanger F, Nicklen S, Coulson AR. DNA sequencing with chain-terminating inhibitors. Proc Natl Acad Sci USA 1977; 74:5463-7.

20 Kozak $M$. An analysis of $5^{\prime}$-noncoding sequences from 699 vertebrate messenger RNA's. Nucleic Acids Res 1987; 15:8125-31.

21 Chan AM-L, McGovern ES, Catalano G, et al. Expression cDNA cloning of a novel oncogene with sequence similarity to regulators of small GTP-binding proteins. Oncogene 1994; 9:1057-63.

22 Ron $\mathrm{D}$, Zannini $\mathrm{M}$, Lewis $\mathrm{M}$, et al. A region of proto- $d b l$ essential for its transforming activity shows sequence similarity to a yeast cell cycle gene $c d c 24$ and 
the human breakpoint cluster gene bcr. New Biol 1991; $3: 372$

23 Shou C, Farnsworth CL, Neel B, et al. Molecular cloning of cDNA's encoding a guanine-nucleotide-releasing factor for RAS p21. Nature 1992; 358:351-4.

24 Haslam RJ, Koide HB, et al. Pleckstrin domain homology. Nature 1993; 363:309-10.

25 Tyers M, Rachubinski RA, Stewart MI, et al. Molecular cloning and expression of the major protein kinase C substrate of platelets. Nature 1988; 333:470-3.

$26 \mathrm{Yu} \mathrm{H}$, Chen JK, Feng S, et al. Structural basis for the binding of proline-rich peptides to $\mathrm{SH} 3$ domains. Cell 1994; 76:933-45.

27 Sloat BF, Adams A, Pringle JR. Roles of $c d c 24$ gene product in cellular morphogenesis during the Saccharomyces cerevisiae cell cycle. F Cell Biol 1981; 89:395405.

28 Hart MJ, Eva A, Evans T, et al. Catalysis of guanine nucleotide exchange on the $\mathrm{CDC} 42 \mathrm{Hs}$ protein by the dbl oncogene product. Nature 1991; 354:311-4.

29 Zheng Y, Olson MF, Hall A, et al. Direct involvement of the small GTP-binding protein RHO in $l b c$ oncogene function. F. Biol Chem 1995; 270:9031-4.

30 Musacchio A, Gibson T, Rice P, et al. The PH domain: a common piece in the structural patchwork of signalling proteins. M Trends Biol Sci 1993; 18:343-8.

31 Koch WJ, Inglese J, Stone WC, et al. The binding site for the beta-gamma subunits of heterotrimeric $G$ proteins on the beta-adrenergic receptor kinase. $f \mathrm{Biol}$ Chem 1993; 268:8256-60.

32 Touhara K, Inglese J, Pitcher JA, et al. Binding of G-protein beta-gamma subunits to plekstrin homology domains. F Biol Chem 1994; 269:10217-20.
33 Harlan JE, Hajduk PJ, Yoon HS, et al. Pleckstrin homology domains bind to phosphatidylinositol-4,5bisphosphate. Nature 1994; 371:168-70.

34 Ron D, Tronick SR, Aaronson SA, et al. Molecular cloning and characterisation of the human $d b l$ proto-oncogene: evidence that its overexpression is sufficient to transform NIH3T3 cells. EMBO f 1988; 7:2465-73.

35 Horri Y, Beeler JF, Sakaguchi K, et al. A novel oncogene, ost, encodes a guanine nucleotide exchange factor that potentially links RHO and RAC signalling pathways. EMBO $\mathcal{F} 1994 ; 13: 4776-86$.

36 Habets GGM, Scholtes EHM, Zuydgeest D, et al. Identification of an invasion-inducing gene, tiam-1, that encodes a protein with homology to GDP-GTP exchangers for RHO-like proteins. Cell 1994; 77:537-49.

37 Hart MJ, Sharma S, elMasry $\mathrm{N}$, et al. Identification of a novel guanine nucleotide exchange factor for the RHO GTPase. I Biol Chem 1996; 271: 25452-8.

38 Bowtell D, Fu P, Simon M, et al. Identification of murine homologues of the Drosophila son of sevenless gene: potential activators of ras. Proc Natl Acad Sci USA 1992; 89:6511-5.

39 Egan SE, Giddings BW, Brooks MW, et al. Association of SOS RAS exchange protein with GRB2 is implicated in tyrosine kinase signal transduction and transformation. Nature 1993; 363:45-51.

40 Rozakis-Adcock M, Femley R, Wade J, et al. The SH2 and $\mathrm{SH} 3$ domains of mammalian GRB2 couple the EGF receptor to the RAS activator mSOS1. Nature 1993; 363:83-5. 


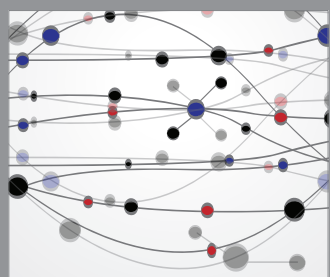

The Scientific World Journal
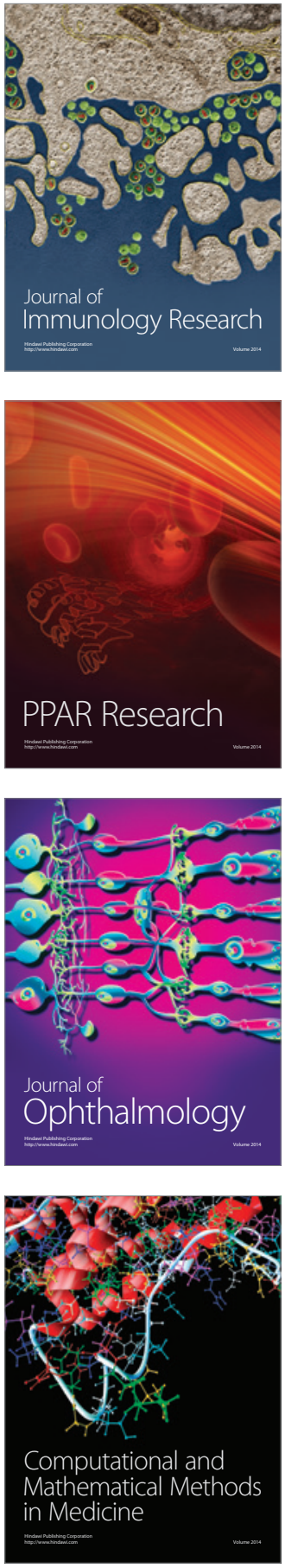

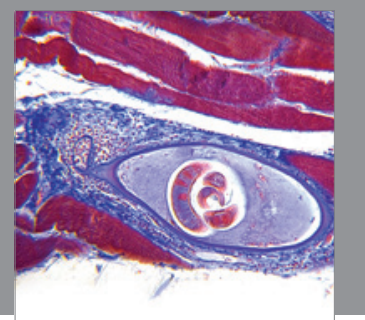

Gastroenterology

Research and Practice
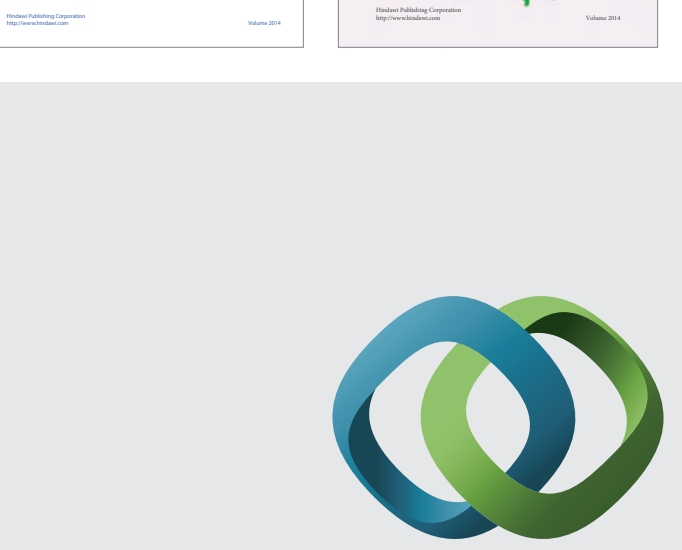

\section{Hindawi}

Submit your manuscripts at

http://www.hindawi.com
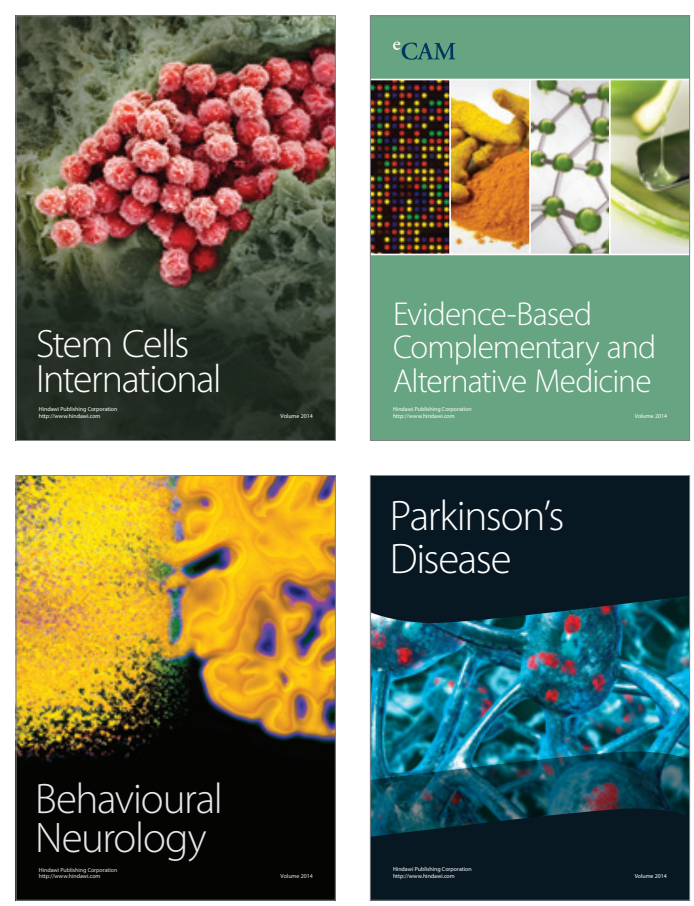

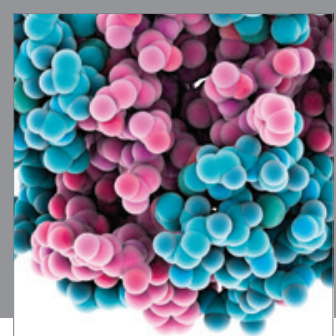

Journal of
Diabetes Research

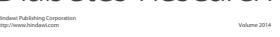

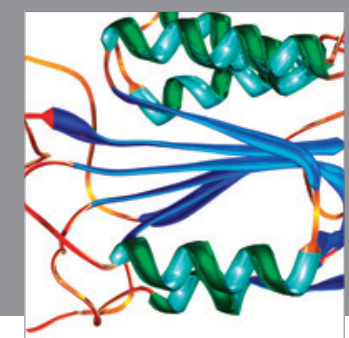

Disease Markers
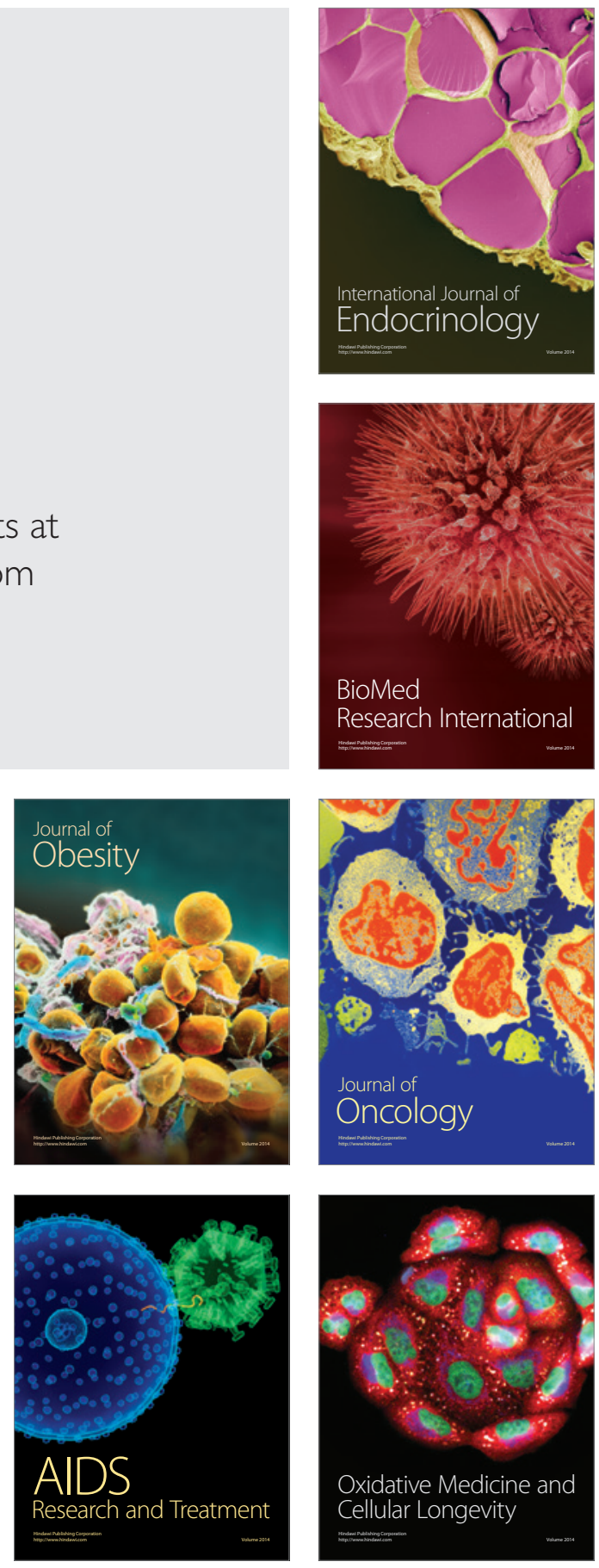